\title{
The evolving role of the laryngeal mask airway in obstetrics
}

\author{
Roanne Preston MD FRCPC
}

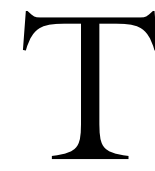

HIS edition of the Journal contains a report that can be considered as astounding in two regards: first, that 1067 healthy women for elective Cesarean section were given a general anesthetic (GA); and second, a laryngeal mask airway (LMA) was used for airway management/ventilation. ${ }^{1}$ Both practices go against the mantra that obstetric anesthesiologists have been teaching for the past 15 years: use regional anesthesia and protect the obstetric airway.

The reasons for this mantra are well-founded. Anesthesia is still a notable cause of maternal mortality. Although the British "Why Mothers Die", published for the triennium 1994-1996, showed a large improvement in anesthesia-related mortality, ${ }^{2}$ Joy Hawkins' 1997 statistics from the USA showed GA to be 16 times more lethal than regional anesthesia in the parturient. ${ }^{3}$ Some of this large difference in risk is definitely attributable to the fact that GA is more likely to be used in emergency situations. It is also true, however, that regional anesthesia is a very safe and effective anesthetic for Cesarean section. The majority of anesthesiarelated maternal deaths worldwide are secondary to aspiration and/or failed intubation. ${ }^{2-4}$ There is no question that endotracheal tubes (ETT) are not the perfect means to prevent aspiration or failure to ventilate indeed the ETT and these two complications are inextricably intertwined. Maternal enquiries consistently find that, in inexperienced hands, the ETT can be fatal. In fact, there is a concern that anesthesiologists are losing their skills in providing general ETT anesthesia in the pregnant woman, and that is contributing to the wide difference in risk attributable to general anesthesia vs regional. Of course, we cannot always use regional anesthesia, and must therefore have the capability and means to provide safe general anesthesia.

In this issue, Han et al. publish the results of their prospective study of 1067 parturients and conclude that "the LMA is effective and probably safe for elective Cesarean section". Why so shocking? Ever since
Mendelson's publication in 1946, where gastric aspiration syndrome was described in parturients undergoing GA with an unprotected airway, ${ }^{5}$ anesthesiologists have been diligent in teaching that protection of the pregnant airway is vital.

The purpose of Han's study was to show that in the hands of experienced operators, the LMA could provide a safe and effective airway in a population classically considered to be at increased risk of aspiration. We know pregnant women have many reasons to have a "full stomach": hormonal changes that effect lower esophageal sphincter tone; gastric acid production; motility changes; the physical effects of the enlarged uterus; and effect of labour pain and narcotics on gastric emptying. Admittedly, the appropriately fasted elective C-section patient with no overt symptoms of reflux is at the low end of the risk scale. But, rather than focussing on absence of reflux/regurgitation/aspiration and ease of ventilation, the authors instead concentrated on minor issues such as ease of insertion, minimal sore throat, and smooth emergence.

The study population was carefully selected: slim (average pregnancy weight of $67 \mathrm{~kg}$ ), fully fasted for six hours, no clinical history of reflux or hiatus hernia, and prophylaxed with antacids. They had optimal anesthesia present upon insertion of the LMA, including the use of muscle relaxation. Cricoid pressure was used, but there is no data on the frequency with which it had to be relaxed to facilitate LMA insertion or ventilation. The prolonged use of cricoid pressure - in this case until delivery - is unlikely to provide any real "protection" against passive reflux. ${ }^{6}$ In $98 \%$ of patients the LMA was inserted on the first attempt, which is an important point to keep in mind. Insertion of a LMA during light anesthesia may predispose to regurgitation and aspiration - the situation one is likely to encounter during a failed intubation/ventilation case. ${ }^{7}$

Han's study shows no clinically apparent aspiration at discharge from the postanesthesia care unit. But, given

From the Department of Anesthesiology, The Ottawa Hospital, Ottawa, Ontario, Canada.

Address correspondence to: Dr. Roanne Preston, Site Chief, Department of Anesthesiology, Director Obstetric Anesthesiology, General Site, The Ottawa Hospital, 501 Smyth Road, Ottawa, Ontario KlH 8L6, Canada. Phone: 613-737-8187; Fax: 613-737-8189;

E-mail: rpreston@ottawahospital.on.ca 
that the main point of this study was to show the LMA to be safe and effective in a non-conventional population, the means of detecting aspiration were very rudimentary: no chest $x$-ray, no $\mathrm{pH}$ probes or dye studies, and no assessment of gastric distension (the study found a $21 \%$ incidence of partially obstructed LMAs). Furthermore, there is minimal specific data on oxygen saturation, end-tidal $\mathrm{CO}_{2}$, or airway pressure. The only parameters of ventilatory "success" were $\mathrm{SpO}_{2}>94 \%$ and $\mathrm{ETCO}_{2}<45 \mathrm{mmHg}$. There is no mention made about how the delivery was facilitated - i.e., did the surgeons exert the usual fundal pressure, which we know in women under regional anesthesia can often precipitate nausea, and/or reflux. In other studies on non-conventional LMA use, intraperitoneal pressures were maintained less than $15 \mathrm{~cm} \mathrm{H}_{2} \mathrm{O}^{8-11}$ How does this measured parameter compare to fundal pressure at delivery?

Previous published incidences of aspiration in obstetrics vary from 1:661 ${ }^{12}$ (Olsson 1986, retrospective, only C- section patients of which $4 / 2623$ aspirated) to 1:1870 13 (Ezri 2000: a retrospective review of 1870 peripartum patients excluding C-section, one case of aspiration; patients were excluded if fasted less than eight hours; average weight was $72 \mathrm{~kg}$ ). Mendelson retrospectively detected an incidence of $1: 667.5$ Statistically, this study has the power to detect clinical aspiration if we accept an incidence of aspiration in the parturient to be greater than 1:1067, which is reasonable given Olsson and Mendelson's data. However, in this study's highly select population, the incidence of aspiration is probably similar to Ezri's study and therefore this study appears to lack adequate power.

Why would one not use regional anesthesia for elective $\mathrm{C}$-section? There are few absolute contraindications, such as patient refusal and overt coagulopathy. For emergency C-section, time pressures often dictate general anesthesia. In a parturient whose airway appears reasonable, and who requires general anesthesia, why would one not use an ETT? Just one serious adverse event from using a device that is not considered to protect the airway, in a parturient deemed to have an easy airway, would be absolutely unacceptable. This study purports that the benefits of a LMA over an ETT include: less hemodynamic stresses, fewer sore throats, better emergence, and fewer changes in pulmonary physiology. Of these, all the authors have shown was that the incidence of sore throat was low at $0.5 \%$. Previous studies have shown that sore throat following LMA use is $5-20 \%$, compared to $25-90 \%$ with an ETT. ${ }^{14}$ Is this an important clinical outcome? If you had a predicted difficult intubation in a parturient and regional anesthesia was contraindicated, would you choose to use a LMA instead of performing an awake intubation with an ETT?
Over the past few years, anesthesiologists have recognized that persisting at endotracheal intubation may lead to increased maternal morbidity and mortality. There is no question that the LMA is proving to be an exceptional anesthesia tool. We have established that the LMA is an acceptable emergency rescue device for the unanticipated difficult intubation in the parturient. ${ }^{15}$ We have accepted that cricoid pressure will probably have to be released to facilitate insertion of the LMA. ${ }^{16}$ The LMA is being used for more and more procedures that were classically considered to be a contraindication to the device either because of the need for muscle relaxation, or because of potentially increased aspiration risk such as with increased intraabdominal pressure during laparoscopic procedures. ${ }^{8-11}$ Most of the studies on such uses of the LMA have been done on a select population: healthy, low risk, no reflux or hiatus hernia, and limitations on body mass index (typically $<30-35$ ). Overwhelmingly, these studies have shown the LMA to be an acceptable alternative to the ETT. Yet, Dr. Brimacombe, author of many articles on the LMA, suggests that for laparoscopic surgery the rule of " 15 ' s" be used if considering a LMA: less than $15^{\circ}$ of Trendelenberg, less than $15 \mathrm{~cm} \mathrm{H}_{2} \mathrm{O}$ intraperitoneal pressure, and a procedure scheduled to last less than 15 min. ${ }^{17}$ Cesarean section does not fit these criteria!

Let us not forget why we first began using an ETT when providing general anesthesia in the parturient:

"Apparently liquid gastric contents were aspirated into the lungs while the laryngeal reflexes were abolished during general anesthesia. The actual aspiration often escaped recognition. Cyanosis, tachycardia, and dyspnea developed as in the obstructive cases, but there was no massive atelectasis. Two patients went on to develop lung abscesses, out of six cases of pneumonia. Two of the five who aspirated solid contents died on the table". 5

After reading Han's study, and having put aside ethical concerns, anesthesiologists should take home only one message: the LMA is acceptable as an emergency device for airway management in the parturient. I would not advocate taking a "the LMA is safe in the maternal airway" message based upon the findings of this study. Remember, these LMAs were inserted in slim, fasted parturients under optimal anesthesia conditions.

What conclusions can we make from this study? In this select population of parturients, the LMA provided a safe and effective airway. If one inserts a LMA for failed intubation in a similar patient, conversion to an ETT is probably unnecessary. We could also reasonably consider using a LMA earlier rather than later following failed endotracheal intubation (perhaps with 
consideration to using the Fastrach or Proseal which have advantages over the classic LMA in terms of airway protection). ${ }^{18}$ But, the standard for airway management in the parturient remains the ETT.

We should not take this study as a sign it is acceptable to return to the practices of the past. We must not erase the great strides that have been made in improving the safety of anesthesia in the parturient by strongly advocating the use of regional anesthesia, and by the establishment of airway management guidelines/difficult airway protocols. Regional anesthesia is still the best choice for the majority of Cesarean sections.

\section{L'évolution du rôle du masque laryngé en obstétrique}

Le présent numéro du Journal renferme un article étonnant pour deux raisons : d'abord, 1067 femmes en bonne santé ont subi une césarienne sous anesthésie générale (AG), puis un masque laryngé (ML) a été utilisé pour la prise en charge des voies aériennes et la ventilation. ${ }^{1}$ Ces deux techniques vont à l'encontre de la règle qu'on enseigne aux anesthésiologistes en obstétrique depuis les 15 dernières années : utiliser l'anesthésie régionale et protéger les voies aériennes.

C'est une règle bien fondée, car l'anesthésie est encore une cause importante de mortalité puerpérale. L'œuvre britannique "Why Mothers Die", publiée pour la période de 1994-1996, a montré une nette régression de la mortalité reliée à l'anesthésie, ${ }^{2}$ mais les statistiques américaines de 1997 de Joy Hawkins ont montré que l'AG est associée 16 fois plus souvent que l'anesthésie régionale au décès des parturientes. ${ }^{3}$ Une partie de cette différence est certainement attribuable au fait qu'on soit plus susceptible d'utiliser l'AG en cas d'urgence. Il est vrai aussi que l'anesthésie régionale est une technique très sûre et efficace pour la césarienne. La majorité des décès puerpéraux reliés à l'anesthésie sont, à travers le monde, secondaires à l'aspiration et/ou à un problème d'intubation..$^{2-4}$ Cela ne fait aucun doute que les tubes endotrachéaux (TET) ne sont pas les meilleurs instruments à utiliser pour prévenir l'aspiration ou la difficulté à ventiler - le TET et ces deux complications sont, en effet, inextricablement liés. Les enquêtes sur les accouchées ont constamment montré que, en des mains inexpertes, l'usage du TET peut être fatal. De fait, on craint que les anesthésiologistes soient moins habiles à utiliser l'anesthésie générale avec TET chez les femmes enceintes et que cela contribue au plus grand risque relié à l'anesthésie générale, comparée à l'anesthésie régionale. On ne peut, bien sûr, utiliser régulièrement l'anesthésie régionale et, en conséquence, on doit pouvoir fournir une anesthésie générale sans risque.

Dans le présent numéro, Han et coll. publient les résultats de leur étude prospective portant sur 1067 parturientes et concluent que "le ML est efficace et probablement sans danger pour la césarienne". Pourquoi cette déclaration est-elle si choquante ? Depuis la publication de Mendelson, en 1946, où on décrit l'aspiration gastrique chez des parturientes qui subissent une anesthésie générale sans protection des voies aériennes ${ }^{5}$, les anesthésiologistes ont tenu à enseigner que la protection des voies respiratoires est vitale chez la femme qui accouche.

L'objectif de l'étude de Han était de montrer qu'en des mains expertes le ML peut permettre une protection sûre et efficace des voies aériennes chez une population habituellement très susceptible d'expérimenter une aspiration gastrique. De nombreuses raisons peuvent expliquer un estomac plein chez une parturiente : des changements hormonaux qui agissent sur le tonus du sphincter inférieur de l'œsophage ; la production d'acide gastrique ; les modifications de la motilité ; les effets physiques de l'augmentation de volume de l'utérus et l'effet de la douleur du travail et des narcotiques sur l'évacuation gastrique. Il faut reconnaître que la patiente à jeun, dont la césarienne a été planifiée et qui ne présente pas de symptômes apparents de reflux se situe au plus bas de l'échelle de risque. Cependant, plutôt que de mettre l'accent sur l'absence de reflux, de régurgitation et d'aspiration, les auteurs se sont concentrés sur des enjeux moins importants comme la facilité d'insertion du tube, le mal de gorge bénin et le réveil en douceur.

La cohorte a été soigneusement sélectionnée : des femmes minces (poids moyen de grossesse de $67 \mathrm{~kg}$ ), complètement à jeun depuis six heures, sans antécédents cliniques de reflux ou de hernie hiatale et qui ont reçu des antiacides préventifs. L'anesthésie a été optimale, depuis l'insertion d'un ML jusqu'à l'usage de myorelaxants. La pression du cricoïde a été utilisée, mais on ne dit rien de la fréquence à laquelle il a fallu la relâcher pour faciliter l'insertion du ML ou la ventilation. L'utilisation prolongée de la pression du cricoïde - dans le cas présent, jusqu'à la naissance du bébé - ne peut sans doute fournir une réelle "protection" contre le reflux passif. ${ }^{6}$ Le ML a été inséré dès le 
premier essai chez $98 \%$ des patientes, ce qui est notable. L'insertion d'un ML pendant une anesthésie légère peut prédisposer à la régurgitation et à l'aspiration - une telle situation étant probable pendant une intubation ou une ventilation impossibles. ${ }^{7}$

L'étude de Han n'a montré aucune aspiration apparente au moment de quitter la salle de réveil. Toutefois, étant donné que le principal objectif de cette étude était de montrer que le ML est sûr et efficace chez une population non classique, les moyens de détection de l'aspiration ont été très rudimentaires : aucune radiographie pulmonaire, aucune mesure du $\mathrm{pH}$, aucune utilisation de colorant et aucune évaluation de distension gastrique (l'étude a trouvé une incidence de $21 \%$ d'obstruction partielle du ML). De plus, il n'y a que des données spécifiques minimales sur la saturation du sang en oxygène, la concentration en $\mathrm{CO}_{2}$ télé-expiratoire ou la pression des voies aériennes. Les seuls paramètres indiquant le "succès" de la ventilation ont été la $\mathrm{SpO}_{2}>94 \%$ et le $\mathrm{CO}_{2}$ téléexpiratoire $<45 \mathrm{mmHg}$. On ne dit rien de ce qui a pu faciliter l'accouchement - c'est-à-dire, est-ce que les chirurgiens ont exercé la pression fundique habituelle, laquelle peut, on le sait, provoquer des nausées et/ou du reflux chez les femmes sous anesthésie régionale. Dans d'autres études sur l'usage non classique du ML, les pressions intrapéritonéales ont été maintenues à moins de $15 \mathrm{~cm} \mathrm{H}_{2} \mathrm{O} .{ }^{8-11}$ Comment peut-on comparer ce paramètre à la pression fundique appliquée pendant l'accouchement ?

L'incidence d'aspiration en obstétrique, publiée antérieurement, varie de $1: 661^{12}$ (Olsson 1986 : rétrospective, césariennes seulement et $4 / 2623$ cas d'aspiration) à $1: 1870^{13}$ (Ezri 2000 : rétrospective de 1870 patientes en péripartum, excluant les césariennes, un cas d'aspiration ; patientes exclues si le jeûne était de moins de huit heures; poids moyen de $72 \mathrm{~kg}$ ). Mendelson a détecté, de façon rétrospective, une incidence de 1 : 667. D' D'un point de vue statistique, l'étude a une puissance suffisante pour détecter l'aspiration clinique si on accepte une incidence de plus de 1 : 1067 chez les parturientes, hypothèse raisonnable selon les données de Olsson et Mendelson. Cependant, chez la population très sélective de la présente étude, l'incidence d'aspiration se rapproche de celle de l'étude de Ezri et, donc, présente une faible puissance statistique.

Pourquoi ne pas utiliser l'anesthésie régionale pour une césarienne ? Il existe peu de contre-indications absolues, comme un refus de la patiente et une coagulopathie apparente. Lors d'une césarienne d'urgence, pressé par le temps, on utilise souvent l'anesthésie générale. Chez une parturiente dont les voies aériennes ne présentent pas de problème apparent, et qui exige une anesthésie générale, pourquoi ne pas utiliser un TET? La survenue d'un seul incident causé par l'usage d'un appareil qui n'est pas considéré comme pouvant protéger les voies aériennes, chez une parturiente jugée facile à intuber, serait absolument inacceptable. L'étude que nous examinons prétend que les bénéfices d'un ML par rapport à un TET comprennent : une réduction du stress hémodynamique et des maux de gorge, un meilleur réveil et moins de changements physiologiques pulmonaires. Tous les auteurs ont montré que l'incidence de mal de gorge était faible, à $0,5 \%$. Des études antérieures ont montré que le mal de gorge suivant l'usage d'un ML est de 5-20\%, comparé à 25-90\% avec le TET. ${ }^{14}$ Est-ce un résultat clinique significatif ? Si vous faites face à une intubation difficile prévue chez une parturiente et que l'anesthésie régionale est contre-indiquée, choisiriez-vous de procéder à une intubation vigile avec un TET ?

Au cours des dernières années, les anesthésiologistes ont reconnu que persister à faire une intubation endotrachéale peut mener à une morbidité et à une mortalité accrue des mères. Il n'y a pas de doute, le ML s'est révélé un exceptionnel outil d'anesthésie. En effet, on a montré que le ML est un dispositif de secours acceptable en urgence dans les cas d'intubation difficile non prévue chez la parturiente. ${ }^{15}$ On a aussi reconnu qu'il faut relâcher la pression du cricoïde pour faciliter l'insertion du ML. ${ }^{16} \mathrm{On}$ utilise de plus en plus le ML pour des interventions considérées auparavant comme une contre-indication à son usage, soit à cause de la nécessité du relâchement musculaire, soit à cause d'un risque d'aspiration potentielle accru comme c'est le cas de la pression intra-abdominale accrue pendant les examens laparoscopiques. ${ }^{8-11}$ La plupart des études sur ces cas d'utilisation du ML ont porté sur une population choisie : en bonne santé, à faible risque, sans reflux ou hernie hiatale et dont l'indice de masse corporelle était dans les limites normales $(<30-35)$. Essentiellement, ces études ont montré que le ML est une solution de rechange acceptable au TET. Néanmoins, le Dr Brimacombe, auteur de nombreux articles sur le ML, recommande l'adoption, pour la chirurgie laparoscopique, de la règle du " 15 ' $\mathrm{s}$ " : une inclination de moins de $15^{\circ}$ en position de Trendelenburg, une pression intrapéritonéale de moins de $15 \mathrm{~cm} \mathrm{H}_{2}$ et une intervention prévue de moins de $15 \mathrm{~min} .{ }^{17} \mathrm{Or}$, la césarienne ne répond à aucun de ces critères !

Rappelons pourquoi le TET a d'abord été utilisé pour l'anesthésie générale chez la parturiente :

"Le contenu gastrique liquide a été aspiré dans les poumons alors que les réflexes laryngés sont abolis pendant l'anesthésie générale. La véritable aspiration peut souvent passer inaperçue. La cyanose, la tachycardie et 
la dyspnée se développent comme dans les cas d'obstruction, mais il n'y a pas d'atélectasie massive. Deux patientes ont été victimes d'abcès pulmonaire, sur six cas de pneumonie. Deux des cinq patientes qui ont aspiré des solides sont mortes sur la table". ${ }^{5}$

Après la lecture de l'étude de Han et la mise à l'écart des préoccupations éthiques, les anesthésiologistes n'ont qu'un message à retenir : le ML est acceptable comme dispositif d'urgence pour la prise en charge des voies aériennes chez la parturiente. D'après les conclusions de cette étude, on ne peut conseiller : "Le ML est sûr pour protéger les voies aériennes d'une parturiente". N'oublions pas que les ML ont été utilisés chez des parturientes minces, à jeun et soumises aux conditions anesthésiques idéales.

Quelles conclusions tirer de cette étude ? Chez la population choisie de parturientes, le ML a permis une protection sûre et efficace des voies aériennes. Si on insère un ML à la suite d'une intubation impossible chez ces patientes, la conversion au TET n'est sans doute pas nécessaire. On peut aussi raisonnablement penser à utiliser un ML plus tôt qu'après l'échec de l'intubation endotrachéale (peut-être le Fastrach ou le Proseal qui présentent des avantages sur le ML traditionnel en regard de la protection des voies respiratoires). ${ }^{18}$ Mais la ligne de conduite normale à suivre pour protéger les voies aériennes chez les parturientes demeure le TET.

Nous ne devons pas voir cette étude comme la caution d'un retour à une pratique du passé. Nous ne devons pas faire fi des grands progrès de l'anesthésie au plan sécurité chez la parturiente en conseillant fortement l'usage de l'anesthésie régionale et en établissant des recommandations de prise en charge des voies aériennes et des protocoles d'intubation difficile. L'anesthésie régionale demeure le meilleur choix pour la majorité des césariennes.

\section{References}

1 Han TH, Brimacombe J, Lee EJ, Yang HS. The laryngeal mask airway for elective Caesarean section in healthy patients. A prospective study of 1,067 cases. Can J Anesth 2001; 48: 1117-21.

2 Department of Health. Why Mothers Die. Report on Confidential Enquiries into Maternal Deaths in the United Kingdom 1994-1996. London: The Stationary Office, Chapter 9, December 1998.

3 Hawkins JL, Koonin LM, Palmer SK, Gibbs CP. Anesthesia-related deaths during obstetric delivery in the United States, 1979-1990. Anesthesiology 1997; 86: 277-84.

4 Dalina $A M$, Inbasegaran $K$ Anaesthetic related maternal deaths in Malaysia - a review. Med J Malaysia
1996; 51: 52-63.

5 Mendelson CL. The aspiration of stomach contents into the lungs during obstetric anesthesia. Am J Obstet Gynecol 1946; 52: 191-205.

6 Brimacombe JR, Berry AM. Cricoid pressure. Can J Anaesth 1997; 44: 414-25.

7 Cheong $\Upsilon-P$, Park $S-K$, Son $\Upsilon$, et al. Comparison of incidence of gastroesophageal reflux and regurgitation associated with timing of removal of the laryngeal mask airway: on appearance of signs of rejection versus after recovery of consciousness. J Clin Anesth 1999; 11: 657-62.

8 Ho BYM, Skinner HJ, Mahajan RP. Gastrooesophageal reflux during day case gynaecological laparoscopy under positive pressure ventilation: laryngeal mask vs. tracheal intubation. Anaesthesia 1998; 53: 910-24.

9 Verghese C, Brimacombe JR Survey of laryngeal mask airway usage in 11,910 patients: safety and efficacy for conventional and nonconventional usage. Anesth Analg 1996; 82: 129-33.

10 Maltby JR, Beriault MT, Watson NC, Fick GH. Gastric distention and ventilation during laparoscopic cholecystectomy: LMA-classic vs. tracheal intubation. Can J Anesth 2000; 47: 622-6.

11 Bapat PP, Verghese C. Laryngeal mask airway and the incidence of regurgitation during gynecological laparoscopies. Anesth Analg 1997; 85: 139-43.

12 Olsson GL, Hallen B, Hambraeus-Jonzon K Aspiration during anaesthesia: a computer-aided study of 185358 anaesthetics. Acta Anaesth Scand 1986; 30: 84-92.

13 Ezri T, Szmuk P, Stein A, Konichezky S, Hagai T, Geva $D$. Peripartum general anaesthesia without tracheal intubation: incidence of aspiration pneumonia. Anaesthesia 2000; 55: 421-6.

14 Joshi GP, Inagaki $\Upsilon$, White PF, et al. Use of the laryngeal mask airway as an alternative to the tracheal tube during ambulatory anesthesia. Anesth Analg 1997; 85: 573-7.

15 Crosby ET, Cooper RM, Douglas MJ, et al. The unanticipated difficult airway with recommendations for management. Can J Anaesth 1998; 45: 757-76.

16 Harry RM, Nolan JP. The use of cricoid pressure with the intubating laryngeal mask. Anaesthesia 1999; 54: 656-9.

17 Brimacombe JR, Berry AM, White PF. The laryngeal mask airway: limitations and controversies. Int Anesthesiol Clin 1998; 36: 155-82.

18 Brimacombe J, Keller C The ProSeal laryngeal mask airway. A randomized, crossover study with the standard laryngeal mask airway in paralyzed, anesthetized patients. Anesthesiology 2000; 93: 104-9. 